\title{
Exposure to trauma and PTSD: findings from the World Mental Health Survey Initiative Portugal
}

\author{
Graça Cardoso ${ }^{1,2}$, Ana Antunes*1,2, Manuela Silva1,2, Sofia Azeredo Lopes ${ }^{3}$, Diana Frasquilho³, Miguel Xavier³, José Miguel \\ Caldas de Almeida ${ }^{1,2}$ \\ ${ }^{1}$ Lisbon Institute of Global Mental Health \\ ${ }^{2}$ Comprehensive Health Research Center, Nova Medical School, Nova University of Lisbon, Lisbon \\ ${ }^{3}$ Nova Medical School, Nova University of Lisbon
}

\section{Background}

Exposure to trauma is common worldwide ${ }^{1}$ but the relative importance of different types of traumatic events in the development of post-traumatic stress disorder (PTSD) varies across countries ${ }^{2,3}$. Given the substantial burden of disability associated with PTSD in Portugal ${ }^{4}$, this study aimed to evaluate exposure to traumatic events, their conditional risk of PTSD and sociodemographic factors associated with traumatic events, lifetime and 12-month prevalence of PTSD.

\section{Materials and Methods}

- Data from the 2009 World Mental Health Survey Initiative Portugal were used $(n=2060)$

- Respondents were inquired about 29 lifetime traumatic events, grouped into 7 categories (war events, sexual violence, accidents, death of a loved one, network events, physical violence, other events)

- The worst traumatic event reported by each participant was used to generate estimates of PTSD associated with each event

- Conditional risk of PTSD was evaluated in relation to each class and type of traumatic event.

- Logistic regression models evaluated the association between sociodemographic factors and exposure to traumatic events, lifetime and 12-month PTSD

Fig 1. Prevalence of lifetime traumatic events in Portugal (\%)

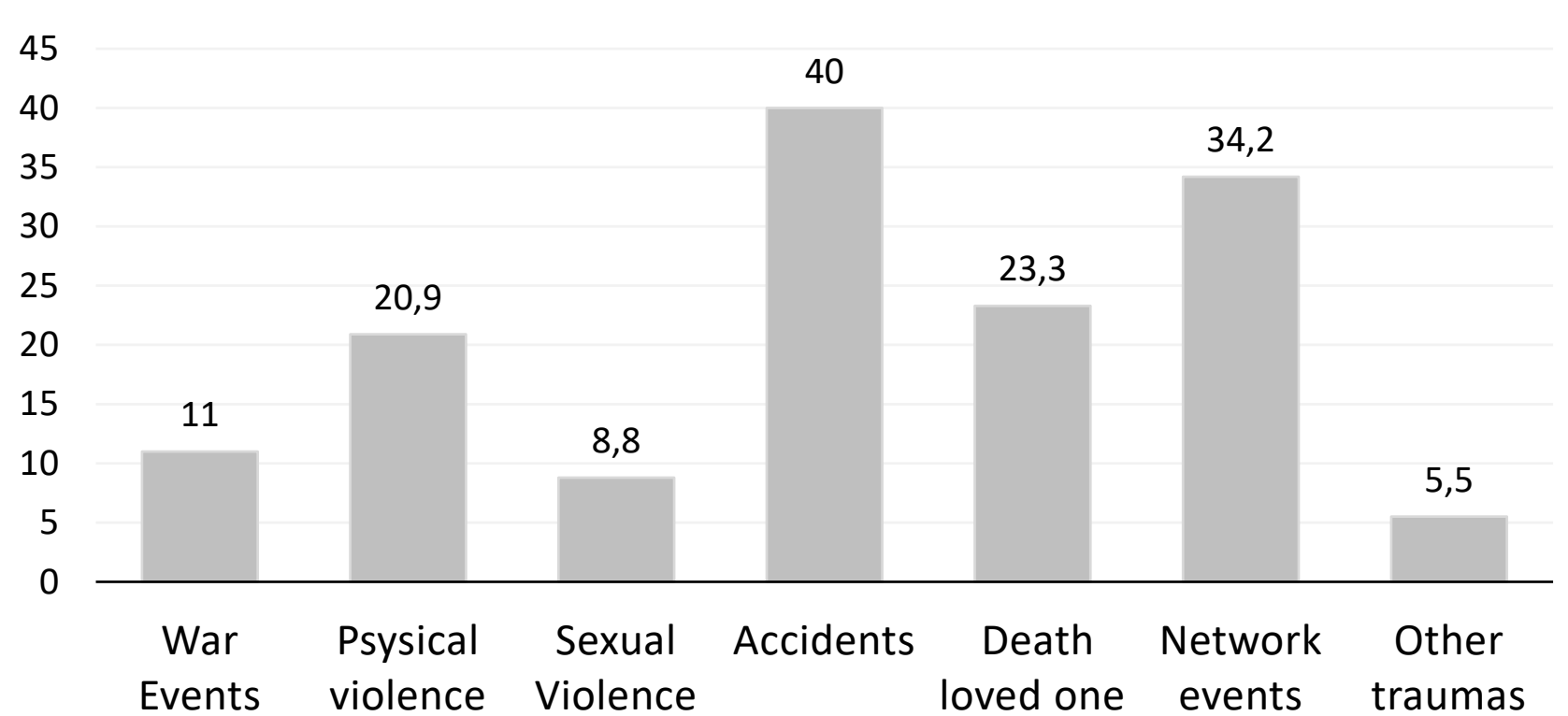

\section{Results}

- Most participants $(69 \% ; n=1548)$ reported at least one lifetime traumatic event

- Having an accident was the class of traumatic events with the highest lifetime prevalence

- Exposure to sexual violence had a low prevalence $(8.8 \%$; s.e. $=0.79)$ compared to other classes of traumatic events but presented the highest conditional risk of PTSD $(19.6 \%$;s.e. $=5.7)$.

- Women were less likely than men to report any lifetime traumatic event (OR=0.6;95\% $\mathrm{Cl}: 0.48-0.80$ )

- After adjusting for type of trauma, women were 2.7 times more likely to experience lifetime PTSD than men (OR=2.7; 95\% Cl:1.47-4.96).

Fig 2. Conditional risk of PTSD (\%) according to classes of traumatic events

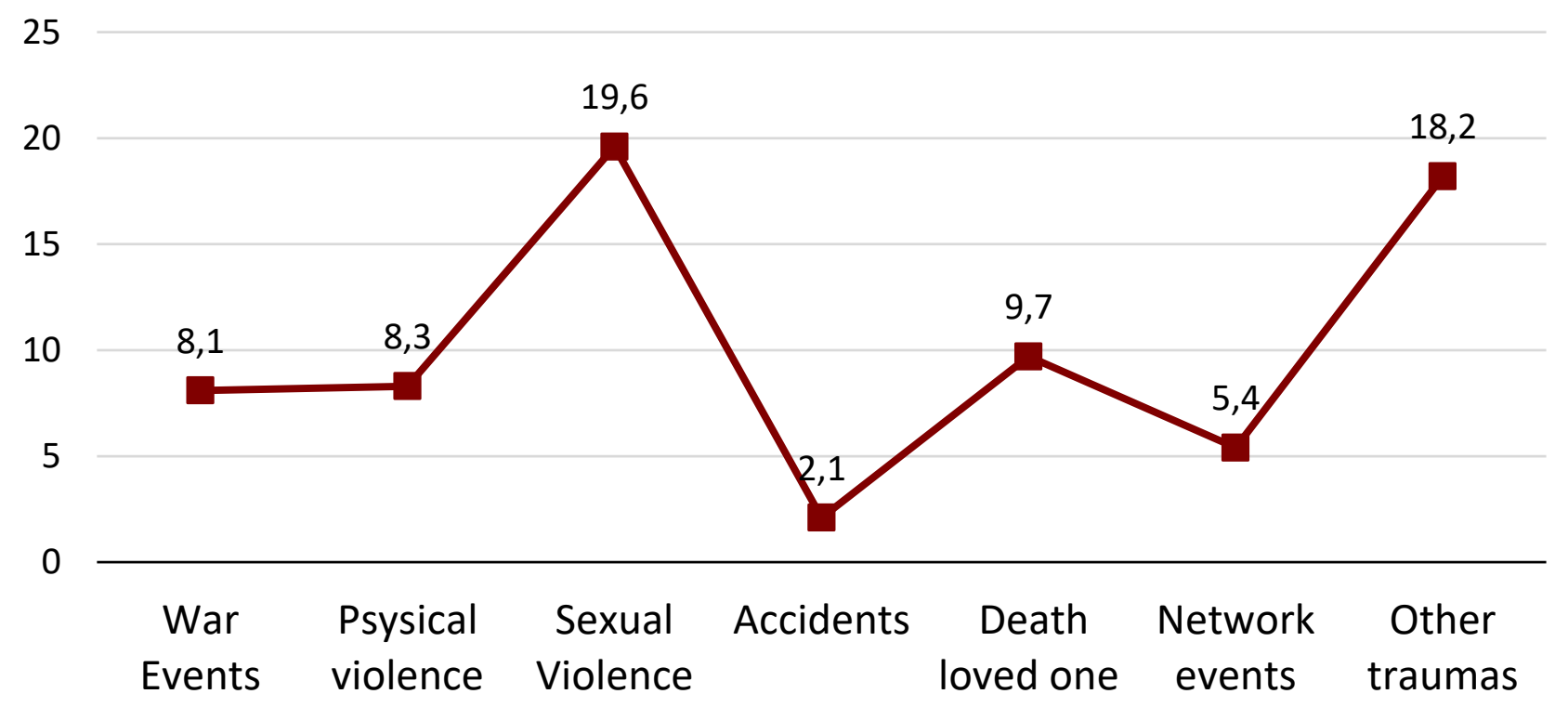

\section{Conclusions}

The findings of this study may contribute to the design of evidence-based interventions aiming to reduce the societal burden of PTSD, drawing attention to the need to recognize sexual violence and gender inequalities as public health priorities in Portugal

\section{References}

1. Benjet, C. et al. 2016. The epidemiology of traumatic event exposure worldwide: Results from the World Mental Health Survey Consortium. Psychol Med. 46(2): 327-343.

2. Kessler, R. et al. 2017. Trauma and PTSD in the WHO world mental health surveys. Eur J of Psychotraumatol 8(5): 1353383.

3. Koenen, K. et al. 2017. Posttraumatic stress disorder in the World Mental Health Surveys. Psychol Med. 47(13): 2260-2274.

4. Antunes, A. et al. 2018. Disability and common mental disorders: Results from the World Mental Health Survey Initiative Portugal. Eur Psychiatry. $49: 56-61$. 